\title{
Measurement of Ductile Forming Limit in Non-linear Strain Paths and Anisotropic Yield Conditions for $11 \%$ Cr Steel Sheets
}

\author{
Takaaki IGUCHI' and Jun YANAGIMOTO \\ 1) Steel Research Laboratory, JFE Steel Corporation, 1 Kawasaki-cho, Chuo-ku, Chiba 260-0835 Japan. \\ 2) Institute of Industrial Science, The University of Tokyo, 4-6-1 Komaba, Meguro, Tokyo 153-8505 Japan.
}

(Received on July 13, 2006; accepted on September 14, 2006)

\begin{abstract}
The ductile forming limit in nonlinear strain paths is investigated for a $11 \% \mathrm{Cr}$ steel which displays a high $r$-value and is used practically in difficult forming applications. Experimental measurements were performed to examine the forming limit in strain paths which involve plane strain loading in first-stage loading and loading at various strain ratios in the second stage. Because the forming limit diagram (FLD) is not applicable to nonlinear loading paths, the forming limit stress diagram (FLSD) theory is investigated. In order to evaluate the stress at the forming limit from measured strain, the anisotropic yield criterion of the material is investigated by uniaxial tension testing in three directions and biaxial stretching using hydraulic bulging. Hill quadratic yield criterion gives the best approximation for biaxial tension, whereas Hosford criterion is more appropriate for uniaxial tension. Yld2000-2d is the ideal criterion for both stress states. Considering the possible effect of dependency of $r$-values on measured strain, the evolution of $r$-values is measured. Under sufficiently large strain, the $r$-value becomes virtually constant. Based on the preliminary investigation outlined above, it was found that the evaluated forming limit stresses in nonlinear strain paths lie on one consistent line in the principal stress field. The limit stress line is not affected by changes in the amount of strain applied in the first loading stage, demonstrating that the FLSD theory of ductile fracture is adequate for this particular material.
\end{abstract}

KEY WORDS: $11 \% \mathrm{Cr}$ steel; sheet forming; forming limit; yield criterion; anisotropy.

\section{Introduction}

It is essential to predict the forming limit of materials in thin sheet forming processes such as stamping in order to design the shapes of products and process parameters. The forming limit diagram (FLD) has been the most commonly used method for this purpose from an early stage of research. The FLD method is based on the fundamental assumptions that fracture occurs in a ductile manner and the loading path is linear, that is, the ratio between the principal stresses or strains remains constant throughout the forming process. In a strict sense, the state of the constant stress ratio does not always correspond to that of the constant strain ratio. However, under a condition of plane stress like that in thin sheet stamping, and as long as elastic strain and stress are not considered, the two states can usually be considered identical. Therefore, in this paper, linear (or proportional) loading refers to both states.

Although the FLD method has proven to be useful for simple stamping processes, it is well known that the FLD is not applicable to nonlinear loading. ${ }^{1,2)}$ This problem appears most evidently in multi-stage forming processes involving drawing, redrawing, and re-striking, where the principal strain ratio in each stage is completely independent from that in the other stages. Inapplicability of the FLD method can also become an issue when the shape to be formed is very complex and in complex loading processes such as tube hydroforming, which is a process involving hydraulic bulging with axial feeding. A different approach is necessary to deal with these problems.

Reviewing the theories of the ductile forming limit, one prominent achievement in the purely theoretical realm began with Swift's diffused necking theory ${ }^{3)}$ and Hill's local necking theory. ${ }^{4}$ The basic concept of those theories is that the onset of mechanical instability is considered to be the forming limit, and this has been accepted and followed by many succeeding researchers. Stören and Rice ${ }^{5)}$ proposed a more elaborate theory based on the bifurcation of the mathematical solution of the system by introducing a yield locus which has a vertex point. The constitutive law which involve the vertex point on the yield locus allows non-unique solutions for succeeding strain increments from a certain stress condition and the bifurcation of the solution. Other types of yield locus involving the vertex point have been proposed by Christoffersen and Hutchinson ${ }^{6}$ ) as the J2 corner theory and by Gotoh ${ }^{7-11)}$ as the J2G theory. Goya and Ito $^{12,13)}$ have proposed a constitutive law which has no vertex point on the yield locus, but allows non-unique solutions for a strain increment which is proportional to the vector of the stress increment. These various theories have been materialized in finite element codes and applied to solve certain practical stamping problems, ${ }^{14-16)}$ but nevertheless, these methods are still not widely used. The major drawbacks are that complexity of the mathematical algebra 
and difficulty of actual implementation in codes. In addition, although the existence of a vertex point on the yield locus has been confirmed experimentally, ${ }^{17}$ the vertex yield locus makes numerical simulation unstable and time consuming.

A completely different approach which is based on void growth theory has been investigated. Microscopic observation has demonstrated that micro-voids exist and grow when tensile strain increases near the ductile fracture point. This theory finally employs one scalar parameter which consists of an integral of some kind of combination of stresses or strains. Fracture is judged by comparing the scalar parameter with a predetermined threshold. Various types of integral functions have been proposed. Takuda et $a l^{18,19)}$ compared and verified some of these functions numerically and experimentally. An interesting point is that, although this approach basically originated from a different viewpoint from the above-mentioned instability theory, both lead to similar forming limit predictions. However, changes in the forming limit in multi-stage forming and nonlinear loading paths have not been discussed thus far. Another problem is that the integral always contains terms of stress, and this cannot be measured easily at the actual production site.

One promising method on which the authors are focusing is the stress-based forming limit theory. When forming limits are plotted on a principal stress field instead of a principal strain field, the limits lie on one curve regardless of their loading path histories. In this paper, this type of stress field or limit curve diagram is called a forming limit stress diagram (FLSD). As attractive features, it is only necessary to determine one FLSD for one material in linear loading paths, and the limit is valid for any nonlinear loading path. Versions of this theory have been proposed by many researchers. $^{20-23)}$

Despite its simplicity and usefulness, the FLSD method has not been widely accepted. One reason may be that the concept that the ductile fracture always occurs at a particular level of stress state is not derived from any theoretical background but comes from series of empirical facts. Actually some cases where the theory is not applicable has been recently reported. ${ }^{24)}$

Another reason may be again that the difficulty of measuring stresses in practical situations, whereas strains can easily be measured by using scribed circles. Recently, Yoshida et $a{ }^{25)}$ verified this theory by measuring stresses experimentally in aluminum tube hydroforming. However, cases where stresses in the material can be measured directly during forming are rare. In general, stresses for fracture prediction must be calculated from actual measured strain or obtained by numerical simulation.

Evaluation of stress from strains employs plastic deformation theory, which requires modeling of a work-hardening rule, a yield criterion, and anisotropic parameters such as the $r$-value of the material concerned. The accuracy of the stresses evaluated by this method will naturally depend on the accuracy of the model, and as long as the model has not been proven to possess sufficient accuracy, the evaluated stresses must be considered to contain discrepancies with respect to the actual values and cannot be used as a general index. On the other hand, experimental corrobora- tion is difficult, as actual stress can only be measured and used to verify the theory in a limited range of cases. ${ }^{24,25)}$

Thus, it is still necessary to confirm if the FLSD method is applicable in every particular situation. In order to do this investigation for the given particular material, the first thing to do is to determine the enough accurate constitutive models such as the yield criterion including the anisotropic behaviors. The next thing is to confirm if the FLSD curves are independent on various nonlinear strain passes.

This research is focused on the above two issues for a particular material which is $11 \% \mathrm{Cr}$ contained steel. The material displays so highly anisotropic behavior, e.g. high $r$ values, that it is suitable for extra deep drawing and complex shape forming applications. In the meantime it is particularly important to determine the accurate constitutive laws, but no report can be seen so far. In particular, the ductile forming limit stress of the material has not been discussed yet. This paper at first presents that this material shows the strain path dependency of the FLD. Next, the suitable yield criterion, the constancy of the anisotropic parameters are presented to evaluate the accurate forming limit stress. Finally the FLSD is evaluated using the verified constitutive laws and its dependency on the strain paths is discussed.

\section{Measurement of FLD for Nonlinear Strain Paths}

The material investigated throughout this research is $11 \% \mathrm{Cr}$ steel, which is classified in AISI409 and for a heat and corrosion resistant use. It contains $11 \% \mathrm{Cr}, 0.2 \% \mathrm{Ti}$ (approx.) and less than $0.03 \%$ C. Specimens for the investigation were taken from cold rolled sheets with a thickness of $1.2 \mathrm{~mm}$.

First, the Forming Limit Diagram (FLD) is measured in 2-stage nonlinear strain paths. The first stage is always plane strain loading, followed by unloading. The secondstage strain paths consist of three types, viz. biaxial, plane strain, and uniaxial loading.

Figure 1 illustrates the method of plane strain loading in the first stage. The specimen size was $80 \mathrm{~mm} \times 200 \mathrm{~mm}$. The longitudinal (L) direction, or rolling direction of the base material, was chosen as the loading direction. The upper and lower sides of the specimen were gripped by wide chucks, and the specimens were stretched at three strain levels, zero strain (i.e., without loading in the first stage) and approximately $10 \%$ or $20 \%$ nominal strain. In order to detect the exact strain induced in this stage, scribed circles of $6 \mathrm{~mm}$ diameter were printed on the specimens be-

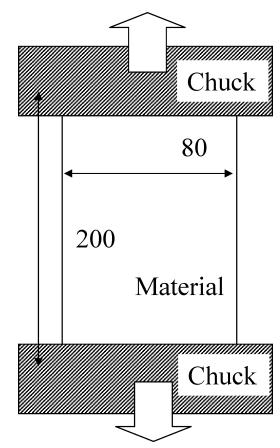

Fig. 1. Method for first-stage loading. 
fore loading, as shown in Fig. 2.

In second-stage loading, biaxial, plane strain, and uniaxial loading were attempted. For the biaxial loading, $50 \mathrm{~mm}$ square specimens were cut from the above-mentioned specimens after first loading, and these were stretched by a spherical punch of $20 \mathrm{~mm}$ diameter, as shown in Fig. 3. For plane strain loading, the first-loaded specimens were cut into rectangles of $33 \mathrm{~mm}$ width $\times 180 \mathrm{~mm}$ length with the slit shape shown in Fig. 4, and tensile tests were performed. The slit shape of the specimen in Fig. 4 was designed referring to the literature. ${ }^{26)}$ For uniaxial loading, JIS13B tensile test specimens were cut from the first-loaded specimens and a tensile test was performed. The tensile direction was the same in the first and second stages in all cases. In the second stage, loading was performed until necking or fracture occurred. In order to evaluate necking and fracture strain, a $2 \mathrm{~mm}$ square mesh pattern was printed on the spec-

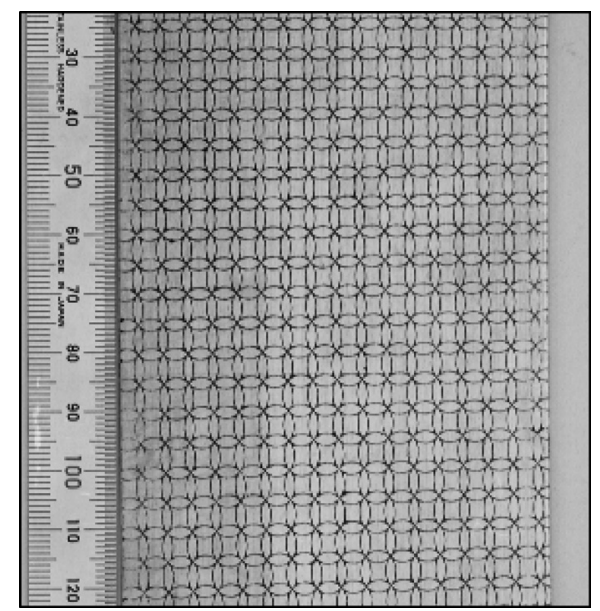

Fig. 2. View of specimen and scribed circles after first-stage loading.

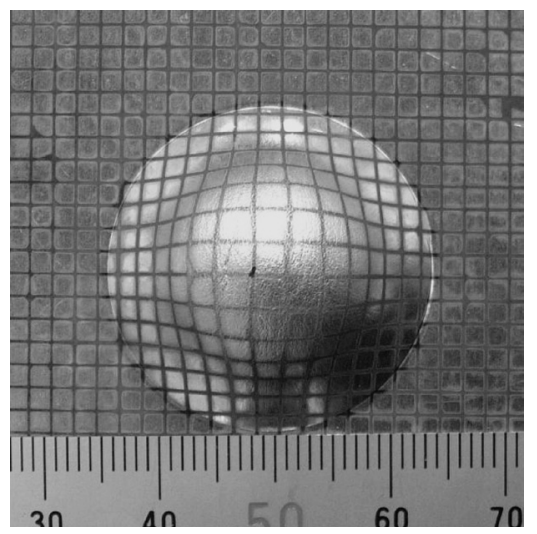

Fig. 3. Upper view of specimen for biaxial stretching test by spherical punch.

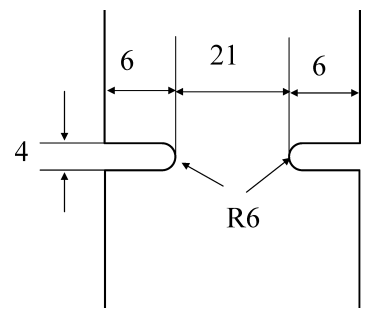

Fig. 4. Dimensions of slits for plane strain tensile test. imens before second-stage loading as shown in Figs. 3 and 5.

For evaluation of the strain in the second stage, in the case of biaxial stretching, loading was stopped at the point when necking was observed, and strain was evaluated at the nearest mesh to the necking point. In the case of plane strain and uniaxial strain, strain was evaluated at the nearest mesh to the fracture points. In this case, the strain is evaluated after the specimen is split completely in two, which means that the fractured portion contains localized necking and subsequent deformation. However, this can lead to overestimation of fracture strain. This problem will be concerned later in this paper.

The two-stage strain paths obtained by the experimental procedure described above are shown in Fig. 6. The strain levels in the first stage consist of three levels, including zero strain. As shown in the figure, when plane strain loading is applied in the first stage, the forming limit curve expands toward the direction of the first principal strain in second-stage loading. Obviously, therefore, the FLD depends on the strain path.

\section{Yield Criterion and Stress-Strain Curve}

As mentioned in the introduction, in order to evaluate stress during deformation from measured strain, an accurate yield criterion and work-hardening model are essential.

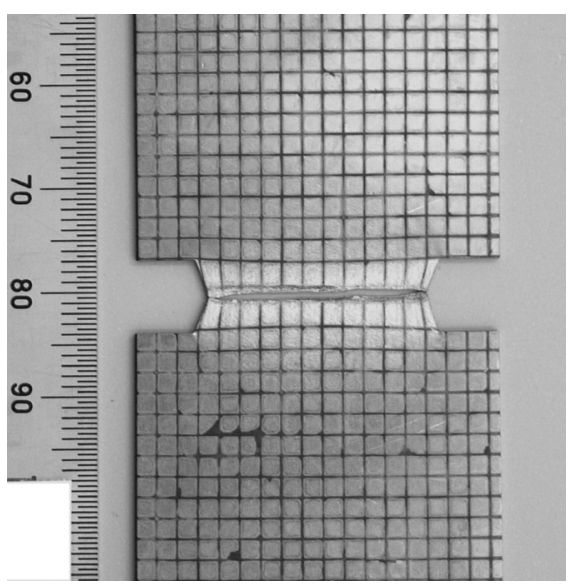

Fig. 5. View of specimen for plane strain tensile test after fracture.

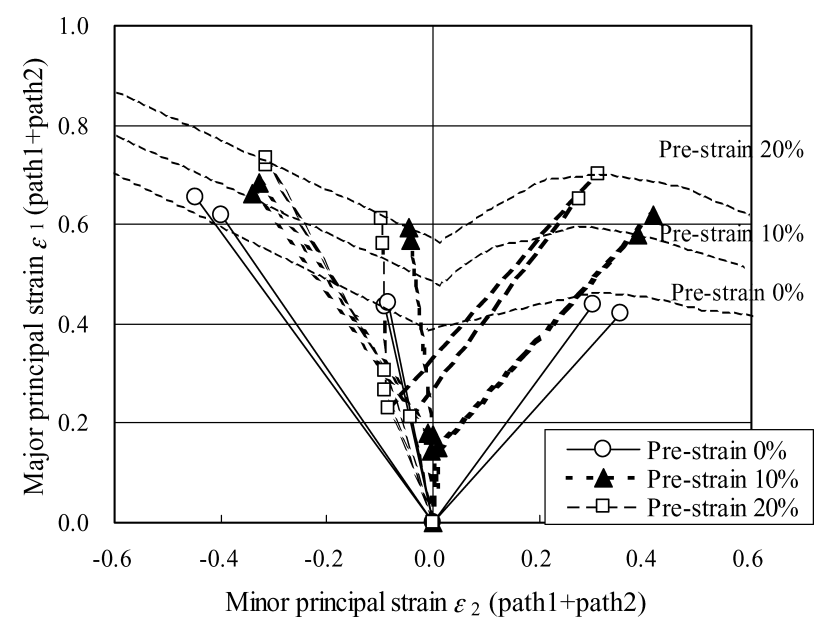

Fig. 6. FLD in nonlinear strain paths (11\% Cr-steel). 


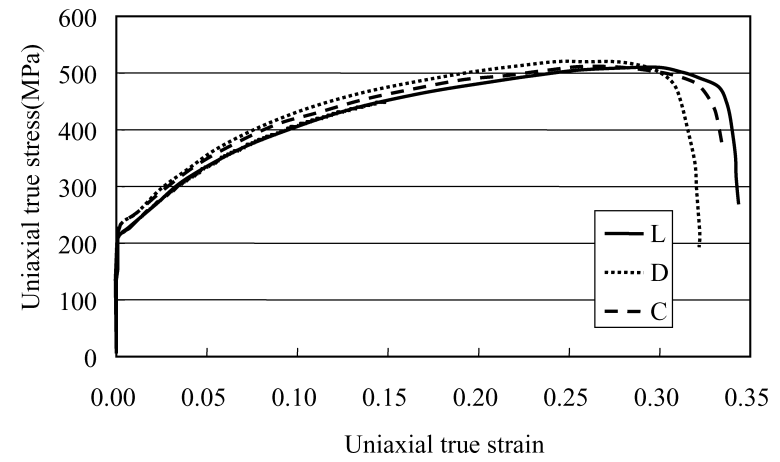

Fig. 7. Results of tensile test of $11 \% \mathrm{Cr}$-steel in each direction.

Therefore, experiments were conducted to verify these items for the material being investigated here. For verification of the yield criterion, the Von-Mises, Hosford, ${ }^{27)}$ and Hill quadratic equations ${ }^{28)}$ are employed.

\subsection{Uniaxial Tension}

First, uniaxial tension tests were performed using JIS13B specimens in the longitudinal (L), cross-sectional (C), and diagonal (D) directions with respect to the rolling direction of the base material. The stress-strain (S-S) curves obtained are shown in Fig. 7. The true logarithmic strain and true stress were evaluated here in such a way that the crosssectional area is calculated based on the assumption of uniform strain and the constant volume law. This means that the stress value is no longer physically valid when it passes over its maximum point. The $r$-values of the material were evaluated by the measured width and thickness of the specimens after stretching at $15 \%$ nominal strain. The $r$-values in the respective directions are 1.535 in the L-direction $\left(r_{\mathrm{L}}\right)$, 2.033 in the C-direction $\left(r_{\mathrm{C}}\right)$ and 1.138 in the D-direction $\left(r_{\mathrm{D}}\right)$. The weighed average $r$-value $\bar{r}$ is 1.461 .

Defining the stress $\sigma_{\mathrm{L}}$ and strain $\varepsilon_{\mathrm{L}}$ in the L-direction as identical with the equivalent stress $\bar{\sigma}$ and strain $\bar{\varepsilon}$, respectively, stress $\sigma_{\mathrm{C}}, \sigma_{\mathrm{D}}$, and strain $\varepsilon_{\mathrm{C}}, \varepsilon_{\mathrm{D}}$ in the $\mathrm{C}$ or D direction are expressed in following ways by the respective yield criteria equations:

(i) Von-Mises equation

$$
\begin{aligned}
\sigma_{\mathrm{C}} & =\sigma_{\mathrm{D}}=\sigma_{\mathrm{L}} \\
\varepsilon_{\mathrm{C}} & =\varepsilon_{\mathrm{D}}=\varepsilon_{\mathrm{L}}
\end{aligned}
$$

(ii) Hosford equation

$$
\begin{aligned}
\sigma_{\mathrm{C}} & =\left\{\frac{\left(1+r_{\mathrm{L}}\right) r_{\mathrm{C}}}{\left(1+r_{\mathrm{C}}\right) r_{\mathrm{L}}}\right\}^{1 / a} \sigma_{\mathrm{L}} \\
\varepsilon_{\mathrm{C}} & =\left\{\frac{\left(1+r_{\mathrm{C}}\right) r_{\mathrm{L}}}{\left(1+r_{\mathrm{L}}\right) r_{\mathrm{C}}}\right\}^{1 / a} \varepsilon_{\mathrm{L}} .
\end{aligned}
$$

Here, $a$ is a constant, which is considered to be 6 for a BCC material such as the ferritic steel considered in this paper.

In the case of Hosford equation, consideration of the Ddirection is not possible.

(iii) Hill quadratic equation

$$
\sigma_{\mathrm{C}}=\sqrt{\frac{\left(1+r_{\mathrm{L}}\right) r_{\mathrm{C}}}{\left(1+r_{\mathrm{C}}\right) r_{\mathrm{L}}}} \cdot \sigma_{\mathrm{L}}
$$

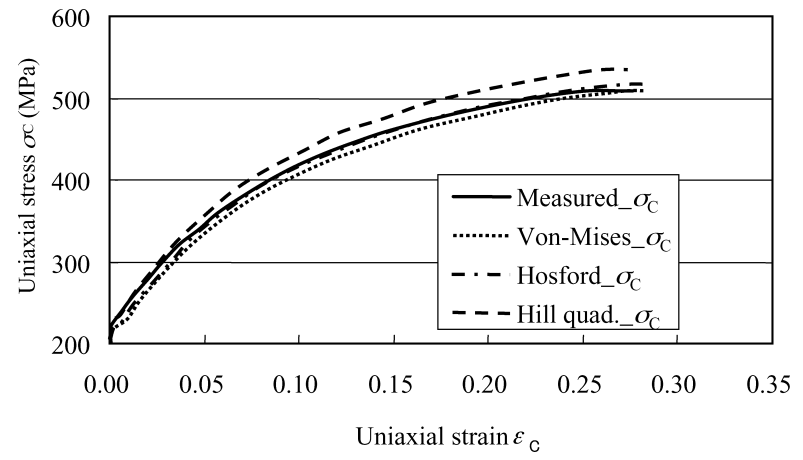

Fig. 8. Comparison between measured and predicted tensile stress in $\mathrm{C}$ direction.

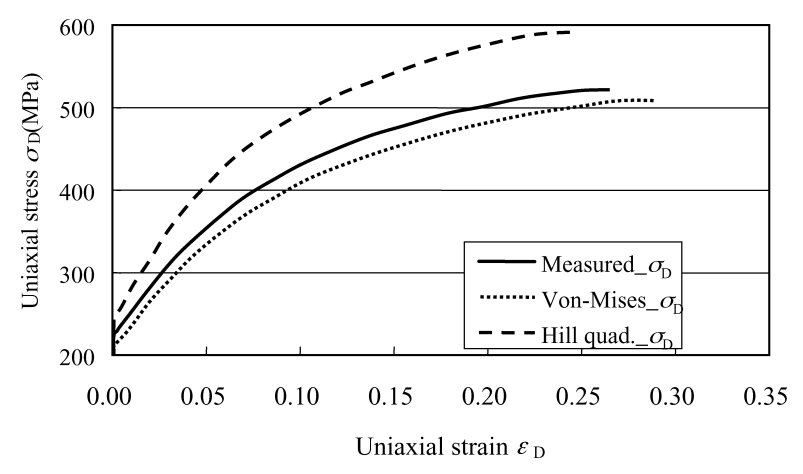

Fig. 9. Comparison between measured and predicted tensile stress in $\mathrm{D}$ direction.

$$
\begin{gathered}
\varepsilon_{\mathrm{C}}=\sqrt{\frac{\left(1+r_{\mathrm{C}}\right) r_{\mathrm{L}}}{\left(1+r_{\mathrm{L}}\right) r_{\mathrm{C}}}} \cdot \varepsilon_{\mathrm{L}} \ldots \ldots \ldots \ldots \ldots \ldots \ldots \ldots \ldots \ldots \ldots \ldots \ldots \\
\sigma_{\mathrm{D}}=\sigma_{\mathrm{L}} / \sqrt{\frac{\left(r_{\mathrm{L}}+r_{\mathrm{C}}\right)\left(1+r_{\mathrm{D}}\right)}{2\left(1+r_{\mathrm{L}}\right) r_{\mathrm{C}}}} \ldots \ldots \ldots \ldots \ldots \ldots \ldots \\
\varepsilon_{\mathrm{D}}=\varepsilon_{\mathrm{L}} \cdot 2\left(1+r_{\mathrm{D}}\right) \\
/ \sqrt{\frac{1+r_{\mathrm{L}}}{r_{\mathrm{L}}}\left(\frac{r_{\mathrm{L}}+r_{\mathrm{C}}+4 r_{\mathrm{L}} r_{\mathrm{C}}}{1+r_{\mathrm{L}}+r_{\mathrm{C}}}+\frac{4 r_{\mathrm{L}} r_{\mathrm{C}}\left(1+2 r_{\mathrm{D}}\right)}{r_{\mathrm{L}}+r_{\mathrm{C}}}\right)} \ldots
\end{gathered}
$$

First, for the C-direction, Fig. 8 shows a comparison between the measured and calculated S-S curves, based on an evaluation by Eqs. (1)-(6) using stress and strain in the Ldirection. Von-Mises and Hill quadratic equations display relatively good agreement between the measured and calculated values. On the other hand, the results given by Hill quadratic equations deviate significantly from the measured results. Specifically, Hill quadratic equation overestimates the anisotropic effect of the $r$-value in the orthogonal direction.

Next, a similar comparison of the S-S curves in the Ddirection is shown in Fig. 9. No curve is shown for Hosford equation because it cannot consider shear components. In this case, the deviation between the measured and calculated results with Hill quadratic equations is much larger than in C-direction. The actual effect of the $r$-value corresponds to the difference between the measured curve and calculated curve by Von-Mises equation, which is overestimated by Hill quadratic equation.

As shown by the measured S-S curves in Fig. 7, the deviation of yield stress levels is small in each direction, al- 


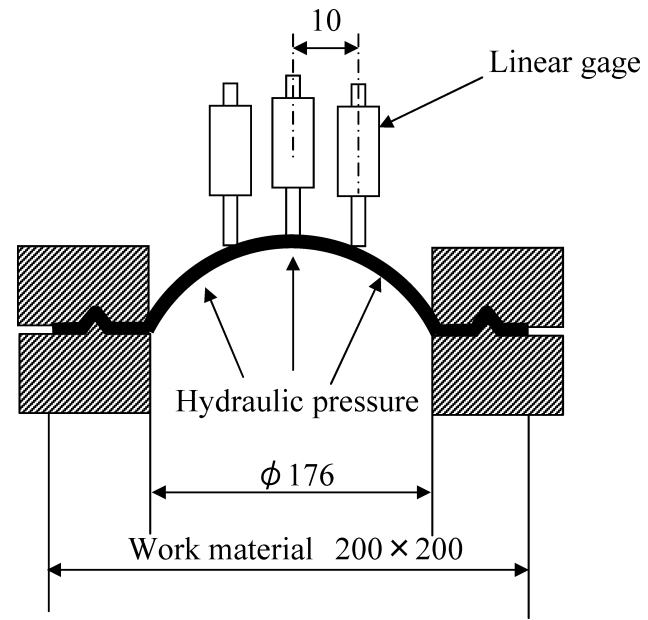

Fig. 10. Method of hydraulic bulge test.

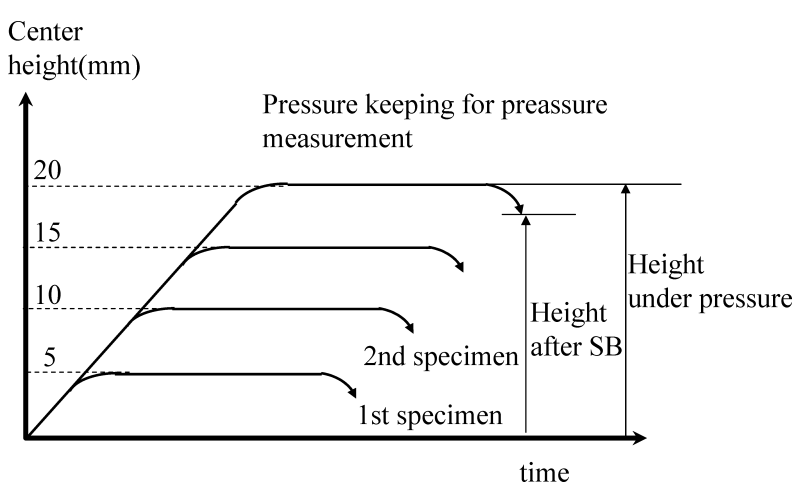

Fig. 11. Method of pressure application.

though the difference in the limit strains is significant. On the other hand, the calculated yield stresses in the C and D directions are higher than that in L-direction due to the higher $r$-values in the $\mathrm{C}$ and $\mathrm{D}$ directions. The measured yield stresses also show the same tendency, but the deviations are much smaller than the estimated results using Hill quadratic yield criterion. Hosford criterion gives better agreement from this viewpoint, but has the drawback that it can handle only the C-direction.

\subsection{Biaxial Tension}

A hydraulic bulge test was performed in order to evaluate yield stresses under biaxial tension. The procedure basically follows Ranta-Eskola's method. ${ }^{29)}$ Specimens $200 \mathrm{~mm}$ square were provided for hydraulic bulge testing, as shown in Fig. 10. Hydraulic pressure is applied within a circular portion of $176 \mathrm{~mm}$ diameter while the surrounding flange is completely fixed by lock beads. Linear gauges are installed vertically at the top center and at points $10 \mathrm{~mm}$ to the left and right sides of center to measure the height $h$ and difference of height $\Delta h$ between the center and the off-centered points. The hydraulic pressure is measured by a pressure sensor installed in the pressure chamber. The thickness change of the dome center is measured by a micrometer in the following manner. Hydraulic pressure is applied to one specimen and kept at a specified level, then unloaded, as shown in Fig. 11, and the thickness of the center of the specimen corresponding to the pressure level is measured. One virgin specimen is used for a test of one pressure level.

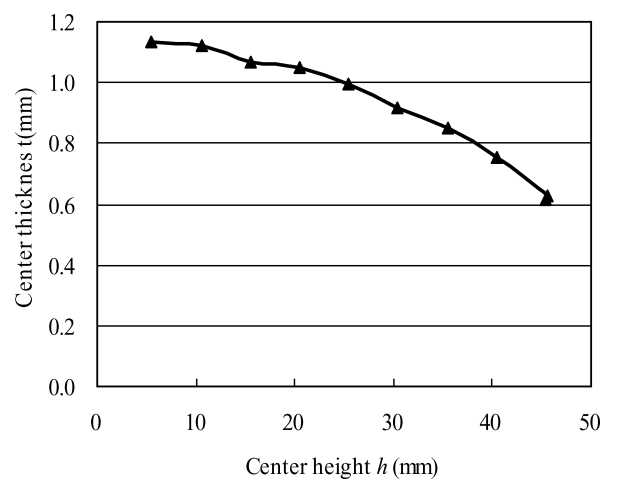

Fig. 12. Curvature $\rho$ measured continuously under loading $(a=10 \mathrm{~mm})$, and measured after unloading $(a=40 \mathrm{~mm})$.

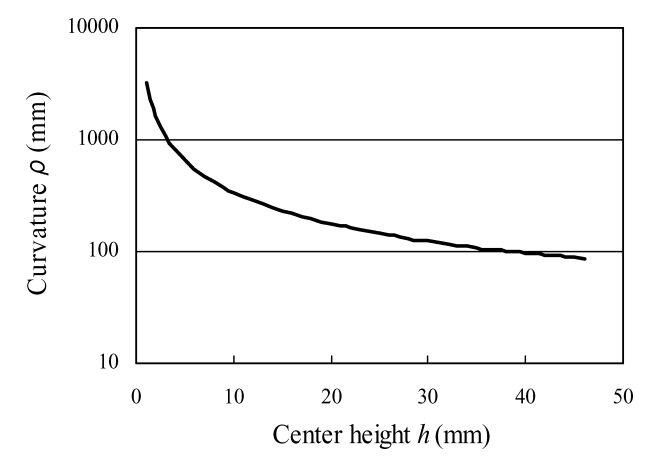

Fig. 13. Relationship between center height $h$ and center thickness $t$.

This procedure is repeated at various pressure levels to obtain a series of relationships between the thickness and pressure. Figure 12 shows the relationship between the measured center height $h$ and the center thickness $t$ of the dome under the unloaded condition. Assuming that this relationship coincides with that in the loaded condition, the thickness under the loaded condition can be interpolated using the height $h$ measured during loading.

The curvature $\rho$ is given by the following equation as an approximate value:

$$
\rho=\frac{a^{2}+\Delta h^{2}}{2 \Delta h}
$$

Figure 13 shows the relationship between the dome height $h$ and the dome curvature $\rho$.

Figure 14 shows the relationship between the dome height $h$ and pressure $P$. Here, the value of $P$ is kept constant, as shown in Fig. 11.

The biaxial yield stress $\sigma_{\mathrm{B}}$ is evaluated from the above measured values using Eq. (10):

$$
\sigma_{\mathrm{B}}=\frac{P \rho}{2 t}
$$

On the other hand, the principal strains $\varepsilon_{1}, \varepsilon_{2}$ are given by:

$$
\varepsilon_{1}=\varepsilon_{2}=\frac{1}{2} \ln \left(\frac{t_{0}}{t_{1}}\right)
$$

The above calculation results in the relationship between the biaxial stress $\sigma_{\mathrm{B}}$ and biaxial strain $\varepsilon_{\mathrm{B}}$ shown by the bold 


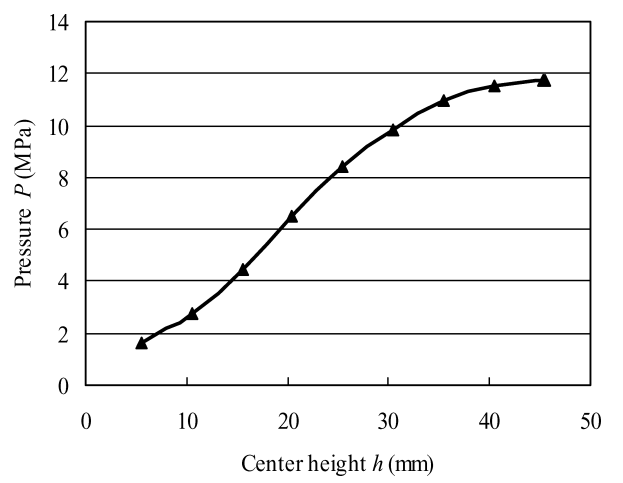

Fig. 14. Relation between center height $h$ and pressure $P$.

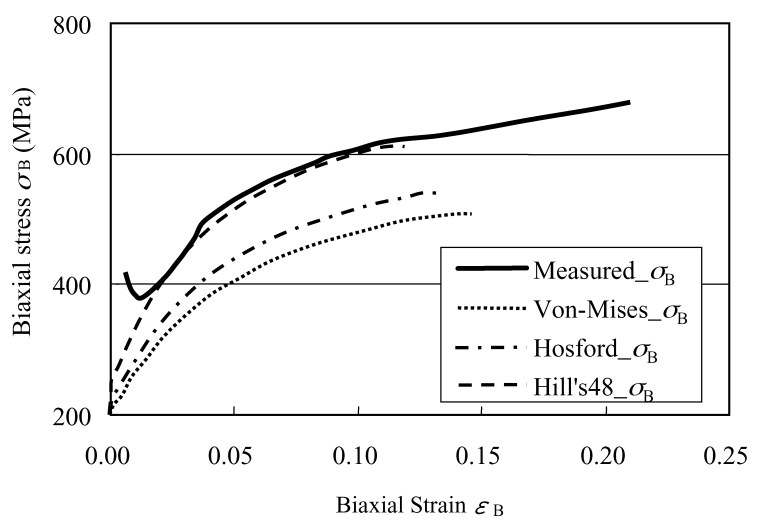

Fig. 15. Comparison between measured and predicted biaxial yield stress $\sigma_{\mathrm{B}}$.

line in Fig. 15.

The calculated values of $\sigma_{\mathrm{B}}$ using the respective yield criteria and the results of the tensile test in the L-direction and $r$-values are shown by the broken lines in Fig. 15. The calculations are performed as follows:

Assuming the stress $\sigma_{\mathrm{L}}$ and strain $\varepsilon_{\mathrm{L}}$ in the L-direction to be equal to the effective stress and strain:

(i) Von Mises equation

$$
\begin{gathered}
\sigma_{\mathrm{B}}=\sigma_{\mathrm{L}} \\
\varepsilon_{\mathrm{B}}=\frac{1}{2} \varepsilon_{\mathrm{L}}
\end{gathered}
$$

(ii) Hosford equation

$$
\begin{aligned}
& \sigma_{\mathrm{B}}=\sigma_{\mathrm{L}}\left(\frac{r_{\mathrm{C}}\left(1+r_{\mathrm{L}}\right)}{r_{\mathrm{L}}\left(1+r_{\mathrm{C}}\right)}\right)^{1 / 6} \\
& \varepsilon_{\mathrm{B}}=2 \varepsilon_{\mathrm{L}}\left(\frac{r_{\mathrm{C}}\left(1+r_{\mathrm{L}}\right)}{r_{\mathrm{L}}\left(1+r_{\mathrm{C}}\right)}\right)^{1 / 6}
\end{aligned}
$$

(iii) Hill quadratic equation

$$
\begin{gathered}
\sigma_{\mathrm{B}}=\sigma_{\mathrm{L}} / \sqrt{\frac{r_{\mathrm{L}}}{1+r_{\mathrm{L}}}\left(\frac{1+r_{\mathrm{L}}}{r_{\mathrm{L}}}+\frac{1+r_{\mathrm{C}}}{r_{\mathrm{C}}}-2\right)} \ldots \ldots . \\
\varepsilon_{\mathrm{B}}=\varepsilon_{\mathrm{L}} / \sqrt{\frac{\left(1+r_{\mathrm{L}}\right) r_{\mathrm{C}}}{\left(1+r_{\mathrm{L}}+r_{\mathrm{C}}\right)}\left(\frac{1+r_{\mathrm{L}}}{r_{\mathrm{L}}}+\frac{1+r_{\mathrm{C}}}{r_{\mathrm{C}}}+2\right)} \ldots
\end{gathered}
$$

Figure 15 shows that the calculation with Hill quadratic equation gives the best agreement with the measured $\sigma_{\mathrm{B}}$ except in an area where the strain is insufficient to ensure the accuracy of measurements. The other yield criteria underestimate the effect of the $r$-value on the biaxial yield stress $\sigma_{\mathrm{B}}$.

Summarizing the above discussion, Hosford yield criterion gives a better approximation of anisotropy in the uniaxial condition. On the other hand, Hill quadratic yield criterion gives the best estimation for biaxial yield stress. Kuwabara et al. ${ }^{30,31)}$ showed that Hosford criterion provides a better approximation at small strain levels of less than $4 \%$ and Hill quadratic criterion gives a better approximation for larger strain levels. Hill quadratic equation is more appropriate because the strain level in this investigation is relatively large, at over $10 \%$. The reference by Kuwabara et $a l^{30)}$ does not show a large discrepancy in uniaxial yield stress in the orthogonal direction, but this is thought to be because the maximum strain was less than $6 \%$ and the $r$ value of the material was not particularly large. For the estimation of the ductile forming limit, the evaluation of the uniaxial strength in some direction is not more important than the biaxial one because fractures in the multi axes stress state are more likely than those in the uniaxial stress state. Taking into account the above discussion, it is understood that Hill quadratic criterion is the most appropriate.

In order to express the yield criterion without contradiction, the Yld2000-2d yield criterion ${ }^{32)}$ which has more variables can be used. The criterion is given as follows:

$$
\left|X_{1}^{\prime}-X_{2}^{\prime}\right|^{M}+\left|2 X_{2}^{\prime \prime}+X_{1}^{\prime \prime}\right|^{M}+\left|2 X_{1}^{\prime \prime}+X_{2}^{\prime \prime}\right|^{M}=2 \bar{\sigma}^{M}
$$

where, $\bar{\sigma}$ is the equivalent yield stress, $M$ is usually taken as 6 for BCC metal as this particular material. $X_{i}^{\prime}$ and $X_{i}^{\prime \prime}$ $(i=1,2)$ are given as follows;

$$
\begin{aligned}
& X_{i}^{\prime}=\frac{1}{2}\left(X_{x x}^{\prime}+X_{y y}^{\prime} \pm \sqrt{\left(X_{x x}^{\prime}-X_{y y}^{\prime}\right)^{2}+4 X_{x y}^{\prime 2}}\right) . \\
& X_{i}^{\prime \prime}=\frac{1}{2}\left(X_{x x}^{\prime \prime}+X_{y y}^{\prime \prime} \pm \sqrt{\left(X_{x x}^{\prime \prime}-X_{y y}^{\prime \prime}\right)^{2}+4 X_{x y}^{\prime \prime 2}}\right) .
\end{aligned}
$$

$X_{x x}^{\prime}, X_{y y}^{\prime}, X_{x y}^{\prime}$ are components of a second rank of tensor $\mathbf{X}^{\prime}$, and $X_{x x}^{\prime \prime}, X_{y y}^{\prime \prime}, X_{x y}^{\prime \prime}$ are components of $\mathbf{X}^{\prime \prime}$. The tensors $\mathbf{X}^{\prime}$ and $\mathbf{X}^{\prime \prime}$ are obtained by the linear translation $\left(\left[X^{\prime}\right]=\left[L^{\prime}\right][\sigma]\right.$, $\left.\left[X^{\prime \prime}\right]=\left[L^{\prime \prime}\right][\sigma]\right)$ of the Cauchy stress tensor $\boldsymbol{\sigma}$ by following tensors $\boldsymbol{L}^{\prime}, \boldsymbol{L}^{\prime \prime}$.

$$
\begin{gathered}
{\left[\begin{array}{l}
L_{11}^{\prime} \\
L_{12}^{\prime} \\
L_{21}^{\prime} \\
L_{22}^{\prime} \\
L_{66}^{\prime}
\end{array}\right]=\left[\begin{array}{ccc}
2 / 3 & 0 & 0 \\
-1 / 3 & 0 & 0 \\
0 & -1 / 3 & 0 \\
0 & 2 / 3 & 0 \\
0 & 0 & 1
\end{array}\right]\left[\begin{array}{l}
\alpha_{1} \\
\alpha_{2} \\
\alpha_{7}
\end{array}\right] \ldots \ldots \ldots \ldots . .(21)} \\
{\left[\begin{array}{l}
L_{11}^{\prime \prime} \\
L_{12}^{\prime \prime} \\
L_{21}^{\prime \prime} \\
L_{22}^{\prime \prime} \\
L_{66}^{\prime \prime}
\end{array}\right]=\frac{1}{9}\left[\begin{array}{ccccc}
-2 & 2 & 8 & -2 & 0 \\
1 & -4 & -4 & 4 & 0 \\
4 & -4 & -4 & 1 & 0 \\
-2 & 8 & 2 & -2 & 0 \\
0 & 0 & 0 & 0 & 9
\end{array}\right]\left[\begin{array}{l}
\alpha_{3} \\
\alpha_{4} \\
\alpha_{5} \\
\alpha_{6} \\
\alpha_{8}
\end{array}\right] \ldots \ldots .(22)}
\end{gathered}
$$


where, $\alpha_{1}-\alpha_{8}$ are constants of Yld2000-2d criterion which are needed to determined by the measurement. The method for the determination of $\alpha_{1}-\alpha_{8}$ is not described here because it requires quite complex algebra and the solution of non-linear simultaneous equations. (For detail see Ref. 31).) Anisotropic parameters which are needed for the Yld2000$2 \mathrm{~d}$ criterion for the material being investigated are shown in Table 1. The stress ratios in Table 1 are evaluated at the points where the nominal strain is $15 \%$, as the $r$-values are. Calculated yield function coefficient $\alpha_{1}-\alpha_{8}$ are listed in Table 2. Here, the assumption $L_{12}^{\prime \prime}=L_{21}^{\prime \prime}$ is employed. The comparison between measured and calculated uniaxial $\mathrm{S}-\mathrm{S}$ curves in $\mathrm{C}, \mathrm{D}$ and biaxial direction from the $\mathrm{S}-\mathrm{S}$ curve in L direction using the Yld2000-2d are shown respectively in Figs. 16, 17 and 18. All of the results show good agreements but it is trivial because the number of unknown coefficients is identical with the number of given anisotropic parameters in this case.

As described above, it is ideal to employ the Yld2000-2d function to express the yield criterion of the material. However, Hill quadratic criterion can be used if the complexity of Yld2000-2d has to be avoided in calculating procedures.

\section{Evolution of $r$-value}

Next, the constancy of the $r$-value is investigated. Throughout this paper, $r$-values are defined as the values calculated from the width and thickness of tensile test specimens under stretching with $15 \%$ nominal strain. However, if strain dependency influences the $r$-value, this would obviously cause discrepancies between the actual and calculated stresses. Therefore, an experiment was carried out in order to evaluate the evolution of the $r$-value during deformation. Specimens $30 \mathrm{~mm}$ in width with a $2 \mathrm{~mm}$ square mesh printed on the surface were prepared, as shown in Fig. 19. The specimens were stretched at various levels of strain, and distortions of the mesh were measured from enlarged photographs using a digitizer to evaluate the $r$-values at the applied strain levels. The results of the relationship between major strain and the $r$-value in the $\mathrm{L}$ and $\mathrm{C}$ directions is shown in Fig. 20. Each $r$-value is an average for the transversal row of twelve meshes in the longitudinal center position of the specimens.

In cases where strain is very small, a large fluctuation of the $r$-value is observed, probably because a tiny distortion of the mesh has a large effect on the $r$-value in this case. However, when the major principal strain is over $15 \%$, the $r$-value becomes stable, converging on the value measured by the standard method.

Ikeda et $a l .{ }^{33)}$ measured the evolution of the $r$-value according to strain for ultra-low carbon IF mild steel. Their

Table 1. Anisotropic parameters of the material.

\begin{tabular}{llllll}
\hline$\sigma_{\mathrm{C}} / \sigma_{\mathrm{L}}$ & $\sigma_{\mathrm{D}} / \sigma_{\mathrm{L}}$ & $\sigma_{\mathrm{B}} / \sigma_{\mathrm{L}}$ & $r_{\mathrm{L}}$ & $r_{\mathrm{C}}$ & $r_{\mathrm{D}}$ \\
\hline 1.016 & 1.041 & 1.215 & 1.535 & 2.033 & 1.138 \\
\hline
\end{tabular}

experiment shows lower $r$-values when the strain is very small and an almost constant value when the strain is larger. Although the behavior in the small strain region is different, the conclusion in the large strain area is the same. However, when discussing forming limits, the strain level is usually high, in most cases being more than $15 \%$. Based on this, we concluded that the $r$-value can be considered to be constant in following discussion.

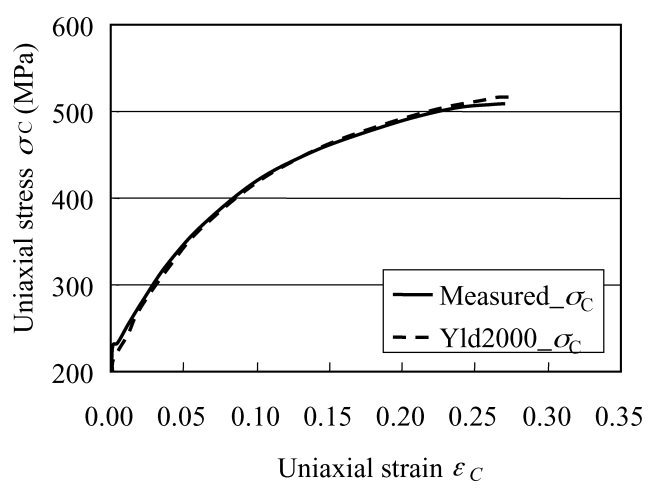

Fig. 16. Comparison between measured and predicted tensile stress by Yld2000-2d in C direction.

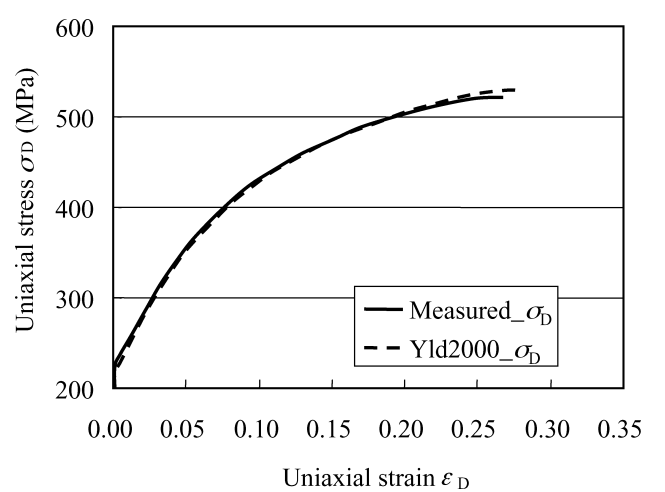

Fig. 17. Comparison between measured and predicted tensile stress by Yld2000-2d in D direction.

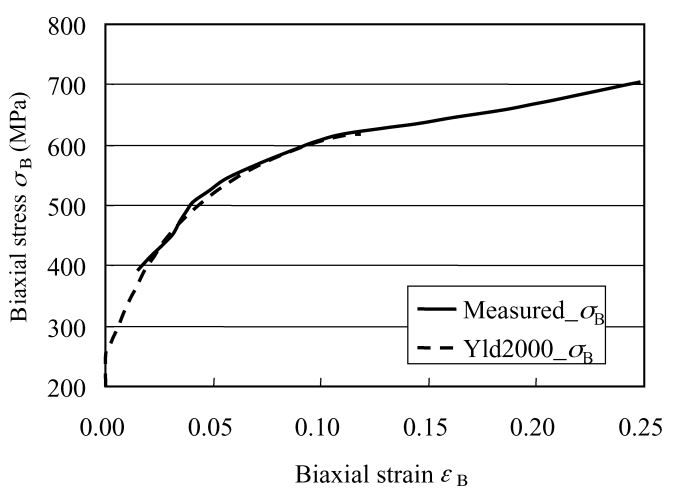

Fig. 18. Comparison between measured and predicted biaxial yield stress by Yld2000-2d.

Table 2. Yield function coefficients of the material.

\begin{tabular}{cccccccc}
\hline$\alpha_{1}$ & $\alpha_{2}$ & $\alpha_{3}$ & $\alpha_{4}$ & $\alpha_{5}$ & $\alpha_{6}$ & $\alpha_{7}$ & $\alpha_{8}$ \\
\hline 0.993 & 1.037 & 0.680 & 0.873 & 0.914 & 0.680 & 0.940 & 1.135 \\
\hline
\end{tabular}




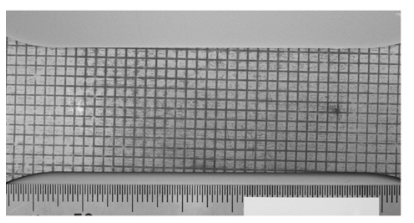

(a) L-direction 2\% stretched

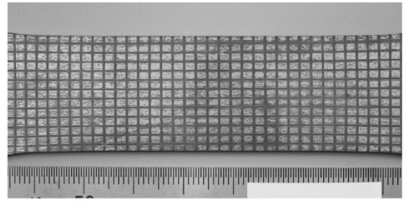

(c) L-direction 14\% stretched
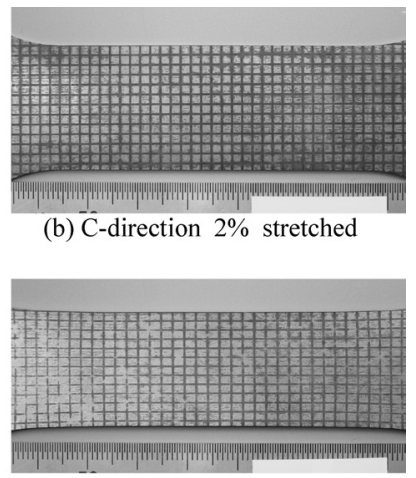

(d) C-direction $14 \%$ stretched (b) $\overrightarrow{\mathrm{C}}$-direction $2 \%$ stretched

Fig. 19. Specimens for continuous measurement of $r$-value.

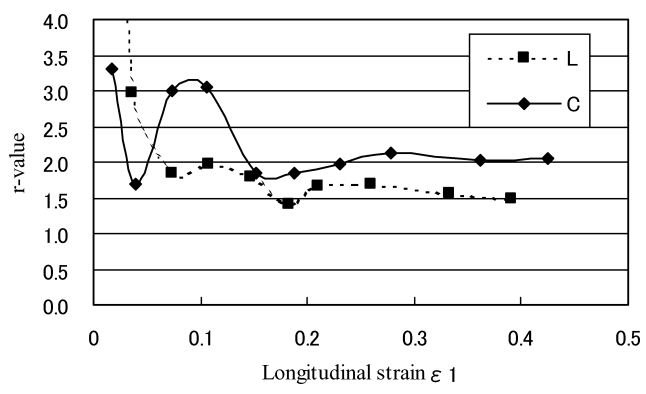

Fig. 20. Measured $r$-value under various strains.

\section{Evaluation of Forming Limit Stress}

Here, we evaluate the forming limit stress diagram (FLSD) from the FLD in the nonlinear strain paths obtained in Fig. 6 using the results presented in Chaps. 2, 3 and 4. The method follows Stoughton. ${ }^{23)}$ First, an S-S curve extending to the very large strain region is necessary. The following equation is employed for the $\mathrm{S}-\mathrm{S}$ curve:

$$
\bar{\sigma}=\sigma_{\mathrm{L}}=F\left(\bar{\varepsilon}+\varepsilon_{0}\right)^{n}
$$

By fitting the unknown parameters of the following equation to the measured S-S curve for the L-direction shown in Fig. 6, we obtain the values of the constants as $F=725.0(\mathrm{MPa}), n=0.25$, and $\varepsilon_{0}=0.001$.

Hill quadratic yield criterion is employed for the reason mentioned in Sec. 3.2 and the convenience for calculations. Considering the case where no shear stress component appears, in other words, in the principal stress condition, the yield condition is expressed as follows:

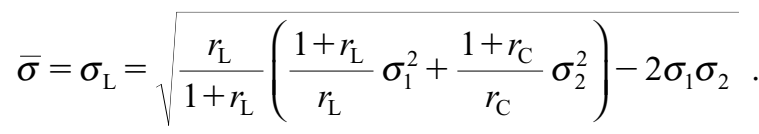

Here, $\sigma_{1}$ is the stress in L-direction and $\sigma_{2}$ is stress in the C-direction.

Using $\alpha=\sigma_{2} / \sigma_{1}$; Eq. (24) is as follows:

$$
\bar{\sigma} / \sigma_{1}=\xi=\sqrt{\frac{r_{\mathrm{L}}}{1+r_{\mathrm{L}}}\left(\frac{1+r_{\mathrm{L}}}{r_{\mathrm{L}}}+\frac{1+r_{\mathrm{C}}}{r_{\mathrm{C}}} \alpha^{2}\right)-2 \alpha}
$$

In the same way, defining $\rho=\dot{\varepsilon}_{2} / \dot{\varepsilon}_{1}$, where $\dot{\varepsilon}_{1}, \dot{\varepsilon}_{2}$ are the principal strain rates in the $\mathrm{L}$ and $\mathrm{C}$ directions, respectively:

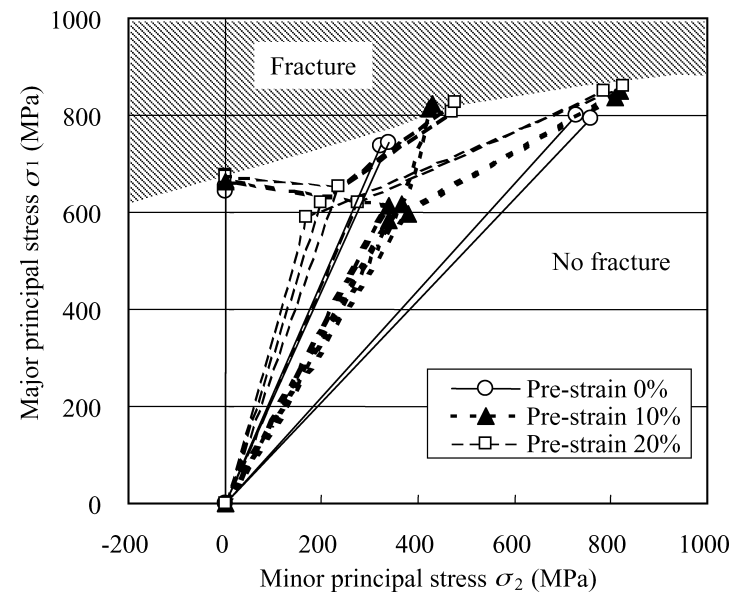

Fig. 21. Forming limit stress diagram.

$$
\dot{\bar{\varepsilon}} / \dot{\varepsilon}_{1}=\lambda=\sqrt{\frac{\left(1+r_{\mathrm{L}}\right) r_{\mathrm{C}}}{1+r_{\mathrm{L}}+r_{\mathrm{C}}}\left(\frac{1+r_{\mathrm{C}}}{r_{\mathrm{C}}}+\frac{1+r_{\mathrm{L}}}{r_{\mathrm{L}}} \rho^{2}+2 \rho\right)} \ldots
$$

The relationship between $\rho$ and $\alpha$ is:

$$
\alpha=\frac{\rho\left(1+\left(1 / r_{\mathrm{L}}\right)\right)+1}{1+\left(1 / r_{\mathrm{C}}\right)+\rho}
$$

We define the major principal strain in the first loading stage as $\varepsilon_{1}^{(1)}$, the strain ratio in this case as $\rho^{(1)}$, the major strain in the second loading stage as $\varepsilon_{1}^{(2)}$, and the strain ratio there as $\rho^{(2)}$. From the above relationships, and assuming that the strain ratio in each loading stage is constant and the strain ratios are identical to the strain rate ratios, the equivalent strain $\varepsilon^{(1)}$, the major principal stress $\sigma_{1}^{(1)}$, and the minor principal stress $\sigma_{2}^{(1)}$ after the first loading stage are calculated as follows:

$$
\begin{gathered}
\bar{\varepsilon}^{(1)}=\lambda^{(1)} \varepsilon_{1}^{(1)}=\lambda\left(\rho^{(1)}\right) \varepsilon_{1}^{(1)} \\
\sigma_{1}^{(1)}=\bar{\sigma}\left(\bar{\varepsilon}^{(1)}\right) \xi\left(\alpha\left(\rho^{(1)}\right)\right) \ldots . \\
\sigma_{2}^{(1)}=\alpha\left(\rho^{(1)}\right) \sigma_{1}^{(1)} \ldots \ldots \ldots . . .
\end{gathered}
$$

Here, $\lambda(\rho), \alpha(\rho)$ and $\xi(\alpha)$ are respectively calculated by Eqs. (26), (27) and (25).

Next, the equivalent strain after the second-stage loading $\overline{\mathcal{E}}^{(2)}$ is evaluated by adding that of the first-stage loading:

$$
\bar{\varepsilon}^{(2)}=\bar{\varepsilon}^{(1)}+\lambda\left(\rho^{(2)}\right) \varepsilon_{1}^{(2)}
$$

The major principal stress $\sigma_{1}^{(2)}$ and the minor principal stress $\sigma_{2}^{(2)}$ in the second loading stage are evaluated using the strain ratio in the second loading stage $\rho^{(2)}=\varepsilon_{2}^{(2)} / \varepsilon_{1}^{(2)}$ as follows:

$$
\begin{gathered}
\sigma_{1}^{(2)}=\bar{\sigma}\left(\bar{\varepsilon}^{(2)}\right) / \xi\left(\alpha\left(\rho^{(2)}\right)\right) . \\
\sigma_{2}^{(2)}=\alpha\left(\rho^{(2)}\right) \sigma_{1}^{(2)} \ldots \ldots . .
\end{gathered}
$$

Using the above equations, the calculated stresses from the strains in Fig. 6 are shown in Fig. 21. Regardless of the change in the strain level in the first loading stage, the limit stresss of forming are plotted on substantially one unique line in the principal stress field. Hence, we can conclude that the theory of the limit stress of forming has been proven to be adequate for this material.

Although this research has demonstrated the above con- 
clusion, it will be necessary to discuss some scattering of the limit stress points from the limit line. The authors believe that this scattering is attributable to the method of measuring the fracture strain, and intend to discuss this problem in the following paper.

\section{Conclusions}

The ductile forming limit in non-linear strain paths was investigated for a functional-type $11 \% \mathrm{Cr}$ steel, which is used in practical applications involving extra deep drawing and complex forming. As the forming limit in a strain field (FLD) is not consistent under the conditions of nonlinear strain paths, the forming limit stress diagram (FLSD) theory was investigated. The anisotropic yield criterion and work-hardening law were investigated in detail, as these must be accurate in order to evaluate the forming limit stress accurately. The results of this research are summarized below:

(1) In the case of uniaxial tension, Hosford yield criterion provides a better approximation for uniaxial stress in the orthogonal direction than Hill quadratic yield criterion. However, in the case of biaxial tension, Hill quadratic yield criterion is more appropriate than Hosford. The ideal criterion for both stress states is Yld2000-2d. However, Hill quadratic criterion is also considered to be usable to evaluate the forming limit.

(2) The $r$-value is not necessarily constant at low strain levels, but converges on a constant value when strain becomes sufficiently large.

(3) The calculated ductile forming limit stresss in complex loading paths, in which plane strain loading is applied in the first stage and loading in various directions is applied in the second stage, lie on one consistent line in the principal stress field, and the limit stress line is not affected by changes in the amount of strain applied in first-loading stage. This result demonstrates that the forming limit stress diagram (FLSD) theory of ductile fracture is adequate for this particular material.

The above achievement enables further investigation to deepen understanding of the forming limit theory. The obtained results of the FLSD for this material can be compared with numerically evaluated ones utilizing the constitutive laws verified in this paper. Other experiments will measure directly the actual stresses at the fracture. and the relationship with obtained limit stresses in this paper will be discussed. Furthermore, verification of the FLSD theory in the case of a forming process involving out-of-plane deformation, which is examined in a similar manner.

The above research will be described in author's subsequent papers.

\section{REFERENCES}

1) K. Nakajima: J. Mater. Process. Technol., 11 (1970), No. 109, 112.

2) H. J. Kleemola and M. T. Pelkkikangas: Sheet Met. Ind., 63 (1977), 591.

3) H. W. Swift: J. Mech. Phys. Solids, 1 (1952), 1.

4) R. Hill: J. Mech. Phys. Solids, 1 (1952), 19.

5) S. Stören and J. R. Rice: J. Mech. Phy. Solids, 23 (1975), 421.

6) J. Christoffersen and J. W. Hutchinson: J. Mech. Phy. Solids, 27 (1979), 465.

7) M. Gotoh: Trans. Jpn. Soc. Mech. Eng. A, 47 (1981), No. 424, 1389

8) M. Gotoh: Trans. Jpn. Soc. Mech. Eng. A, 48 (1982), No. 428, 493.

9) M. Gotoh: Trans. Jpn. Soc. Mech. Eng. A, 48 (1982), No. 428, 502.

10) M. Gotoh: Trans. Jpn. Soc. Mech. Eng. A, 49 (1983), No. 437, 92.

11) M. Gotoh: Trans. Jpn. Soc. Mech. Eng. A, 50 (1984), No. 458, 1753.

12) M. Goya and K. Ito: Trans. Jpn. Soc. Mech. Eng. A, 54 (1988), No. 504, 1617.

13) M. Goya and K. Ito: Trans. Jpn. Soc. Mech. Eng. A, 56 (1990), No. $521,101$.

14) N. Iwata, M. Matsui and M. Gotoh: J. Jpn. Soc. Technol. Plast., 33 (1992), No. 381, 1196.

15) N. Iwata, M. Matsui and M. Gotoh: J. Jpn. Soc. Technol. Plast., 33 (1992), No. 381, 1202.

16) T. Yoshida, K. Ito, Y. Kuriyama and M. Usuda: J. Jpn. Soc. Technol. Plast., 42 (2001), No. 488, 919.

17) T. Kuwabara, M. Kuroda, V. Tvergaard and K. Nomura: Acta Mater, 48 (2000), No. 9, 2071

18) H. Takuda, K. Mori, H. Fujimoto and N. Hatta: Arch. Appl. Mech., 67 (1997), 143.

19) H. Takuda, K. Mori and N. Hatta: J. Mater. Process. Technol., 95 (1999), 116.

20) J. Gronostajski: J. Mech. Work. Technol., 10 (1984), 349.

21) R. Arrieux: Ann. CIRP, 36 (1987), No. 1, 195.

22) L. Zhao, R. Sowerby and M. P. Sklad: Int. J. Mech. Sci., 38 (1996), 1307.

23) T. B. Stoughton: Int. J. Mech. Sci., 42 (2000), 1.

24) K. Yoshida and T. Kuwabara: Tetsu-to-Hagané, 92 (2006), No. 1, 36.

25) K. Yoshida, T. Kuwabara, K. Narihara and S. Takahashi: J. Jpn. Soc. Technol. Plast., 45 (2004), No. 517, 123.

26) R. H. Wagoner: Metall. Trans. A, 12A (1981), 877.

27) R. W. Logan and W. F. Hosford: Int. J. Mech. Sci., 22 (1980), 419.

28) R. Hill: Proc. R. Soc. (London), $\mathbf{A 1 9 3}$ (1948), 281.

29) A. J. Ranta-Eskola: Int. J. Mech. Sci., 21 (1979), 457.

30) T. Kuwabara, S. Yamada, E. Iizuka and T. Hira: Tetsu-to-Hagané, 87 (2001), No. 4, 40.

31) T. Kuwabara and S. Ikeda: Tetsu-to-Hagané, 88 (2002), No. 6, 50.

32) F. Barlat, J. C. Brem, J. W. Yoon, K. Chung, R. E. Dick, D. J. Lege, F. Pourboghrat, S.-H. Choi and E. Chu: Int. J. Plast., 19 (2003), 1297.

33) S. Ikeda and T. Kuwabara: Japanese Spring Conf. for the Technology of Plasticity, JSTP, Tokyo, (2003), 229. 